\title{
Haptic orientation perception benefits from visual experience: Evidence from early-blind, late-blind, and sighted people
}

\author{
Albert Postma \\ Helmholtz Institute, Utrecht University, Utrecht, The Netherlands \\ SANDER ZUIDHOEK \\ Visio Noord-Nederland, Haren, The Netherlands \\ MatThiss L. NoordziJ \\ F. C. Donders Center for Cognitive Neuroimaging, \\ Radboud University Nijmegen, Nijmegen, The Netherlands \\ AND \\ ASTRID M. L. KAPPERS \\ Helmholtz Institute, Utrecht University, Utrecht, The Netherlands
}

\begin{abstract}
Early-blind, late-blind, and blindfolded sighted participants were presented with two haptic allocentric spatial tasks: a parallel-setting task, in an immediate and a 10-sec delay condition, and a task in which the orientation of a single bar was judged verbally. With respect to deviation size, the data suggest that mental visual processing filled a beneficial role in both tasks. In the parallel-setting task, the early blind performed more variably and showed no improvement with delay, whereas the late blind did improve, but less than the sighted did. In the verbal judgment task, both early- and late-blind participants displayed larger deviations than the sighted controls. Differences between the groups were absent or much weaker with respect to the haptic oblique effect, a finding that reinforces the view that this effect is not of visual origin. The role of visual processing mechanisms and visual experience in haptic spatial tasks is discussed.
\end{abstract}

Our haptic sense provides us with essential information about the spatial layout of peripersonal space - that is, the size, shape, position, and orientation of things within reach. Remarkably, haptic perception of basic spatial properties such as line length (Lanca \& Bryant, 1995; Marks \& Armstrong, 1996), path length (Lederman, Klatzky, \& Barber, 1985), and orientation (see, e.g., Appelle \& Countryman, 1986; Gentaz \& Hatwell, 1998, 1999; Kappers, 1999; Zuidhoek, Visser, Bredero, \& Postma, 2004) is susceptible to marked distortions, at least in blindfolded sighted individuals. Interestingly, the distortions in haptic perception of orientation appear to be both systematic over multiple locations and consistent over different tasks - for example, the setting of a test bar either parallel or collinear with a reference bar, or the pointing of a bar toward a marker (Kappers, 1999, 2002; Kappers \& Koenderink, 1999). In the present study, we set out to investigate the nature of these systematic errors further by comparing blind participants to sighted controls.

Kappers (2003) argued that setting two bars parallel to each other in a plane on the basis of touch alone typically recruits a mixture of egocentric (viz., hand-centered) and allocentric reference frames. The effect of the former is indicated by large, systematic errors - that is, clockwise with test bars to the right and counterclockwise with test bars to the left of the reference bar (see, e.g., Kappers, 1999, 2003; Kappers \& Koenderink, 1999; Zuidhoek, Kappers, van der Lubbe, \& Postma, 2003, Zuidhoek et al., 2004). An egocentric reference frame presupposes coding of the locations and spatial orientations of items in the outside world with respect to a part of one's body, such as the hands, the midsagittal plane (Zuidhoek, Kappers, \& Postma, 2005), the body as a whole (Heller, Calcaterra, Green, \& Barnette, 1999), or a subjective gravitational frame (Luyat, Gentaz, Corte, \& Guerraz, 2001). In an allocentric reference frame, spatial information is encoded with respect to external landmarks in the outside world, such as when taking into account the available surrounding background information (Millar, 1988, 1994; Thinus-Blanc \& Gaunet, 1997). The first phase of haptic processing might almost obligatorily involve the employment of an ego- or bodycentered reference frame. It should be noted that in the

A. Postma, a.postma@uu.nl 
parallel-setting task, veridical performance and allocentric performance coincide. Thus, "getting better" by definition means interpreting the haptic orientation within an accurate, allocentric reference frame (see Kappers, 2003).

Interestingly, recent evidence has revealed that visual inputs and visuospatial imagery may ameliorate haptic orientation processing. For example, viewing the region of space directly above the haptic workspace has been found to improve parallel-setting performance (Newport, Rabb, \& Jackson, 2002; Zuidhoek et al., 2004). In addition, in the latter study, the directions of the head and eyes were found to affect parallel-setting performance independently of visual input, with better parallel-setting performance when the head and eyes were directed toward the reference bar, rather than straight ahead or toward the test bar. When multiple cues (in particular, visual background cues) are combined, haptic processing of orientation might be elevated (see Millar \& Al-Attar, 2004). Furthermore, a haptic orientation perception task with a verbal response requiring the translation of the orientation of a single bar into the corresponding number of minutes on an imagined clock (the "verbal judgment of orientation task") has been found to result in very small (yet similarly systematic) deviations, as compared with haptic parallel-setting (Zuidhoek et al., 2005). Also, a marginally significant correlation was found between performance on this task and performance on a visuospatial imagery task (Paivio's [1978] mental clock test). Zuidhoek et al. (2005) argued that response methods stimulating mental imagery of the felt orientation may facilitate an allocentric representation.

Notably, visual information processing does not always need to have beneficial effects in haptic orientation perception tasks. ${ }^{1}$ In a haptic matching task for which an intrinsic or egocentric representation is believed to be sufficient - mirroring with respect to the midsagittal planenoninformative vision was found to have a slightly negative influence on performance (Newport et al., 2002). For parallel-setting, which by definition requires replication of an orientation within allocentric space, a positive effect of noninformative vision has been found instead (Newport et al., 2002; Zuidhoek et al., 2004).

Another finding that bears on the idea that certain circumstances evoke a different interpretation of the haptic inputs is that, in haptic parallel-setting, the retention of a reference bar orientation for $10 \mathrm{sec}$ before rotating the test bar results in smaller deviations in parallel-setting performance than those found for nondelayed performance (Zuidhoek et al., 2003). In line with several visual and proprioceptive goal-directed action studies (Bridgeman, Peery, \& Anand, 1997; Milner, Paulignan, Dijkerman, Michel, \& Jeannerod, 1999; Rossetti, Gaunet, \& Thinus-Blanc, 1996; Rossetti \& Régnier, 1995), we have interpreted this improvement as reflecting a shift from an egocentric toward a more allocentric spatial representation during the delay time.

Together, the studies discussed above suggest that when processing haptic orientation inputs, a shift from a more egocentric to a more allocentric reference frame may occur over time. This shift may further be stimulated by concurrent visual processing. If so, the question arises of the extent to which visual experience is important in performing haptic spatial tasks. Ernest (1987) argued that among the sighted, strategies "are primarily visual and externally derived; in the blind they are predominantly auditory, kinesthetic, and based on internal cues. Indeed the strategies of the blind may be generalized as selfreferent." Consequently, in this view, the sighted could be predicted to outperform both the early and the late blind on tasks requiring an allocentric representation, such as the haptic parallel-setting task or a haptic perception task in which orientation is explicitly allocentrically defined. On a different note, Millar $(1988,1994)$ has argued that preferred strategies depend on whatever reliable information is available to an individual. In many circumstances, blind individuals have to rely almost exclusively on more body-centered proprioceptive and kinesthetic information, causing them difficulty in organizing the spatial relationships between objects into allocentric representations. However, if the cues available to blind individuals increase or if sighted individuals are prevented from accessing external (visual) cues, blind and sighted groups should perform similarly.

In line with the foregoing analysis, the goal of the present study was to further establish the role of visual experience in haptic orientation processing. We had early-blind, late-blind, and blindfolded sighted participants perform two tasks that are thought to differ in the extents to which they stimulate the use of egocentric and allocentric reference frames: the parallel-setting task (Experiment 1; see Kappers, 2003; Newport et al., 2002; Zuidhoek et al., 2003 ) and the verbal judgment task (Experiment 2; see Zuidhoek et al., 2005).

\section{EXPERIMENT 1 Parallel-Setting Task}

Our first task was a haptic parallel-setting task, in which participants were required to haptically match the orientation of a test bar with that of a reference bar. Parallel-setting was performed with 0 - and 10 -sec delays. Zuidhoek et al. (2003) showed that in the 0 -sec delay condition, blindfolded sighted individuals rely to a great extent on an egocentric reference framework, resulting in relatively large distortions of haptic orientation processing. With a 10 -sec delay, performance improves, suggesting a shift to a more allocentric representation. Interestingly, Rossetti et al. (1996) found that, whereas delaying a pointing action in the proprioceptive domain for $8 \mathrm{sec}$ leads to a qualitatively different endpoint distribution pattern in the blindfolded sighted, it does not in the early blind: Although the endpoint distributions of their blindfolded sighted participants showed a transition from alignment with the pointing movement (egocentric) in the immediate condition toward alignment with the arcshaped target array (allocentric) with delay, those of the early blind showed endpoint distributions aligned with the direction of the pointing movement not only in the immediate, but also in the delayed condition, although less distinctly. 


\section{Method}

Design. The experiment had a $2 \times 2 \times 3$ factorial design with delay ( 0 vs. $10 \mathrm{sec})$ and orientation (oblique vs. cardinal) as withinsubjects factors, and group (early blind, late blind, and sighted) as a between-subjects factor. Delay comprised two, blocked conditions - that is, immediate and delayed motor matching of haptically perceived orientation ( 0 - and 10 -sec delay, respectively). Half of the participants started with the immediate condition, and the other half with the delayed condition. Orientation comprised two levels: oblique (including four reference bar orientations) and cardinal (including two reference bar orientations). The various orientations were randomly presented within a delay block.

Participants. All participants were enrolled in an elaborate study on spatial cognition in the blind, in which haptic, verbal, and imagery tasks were conducted on a single day of testing (the verbal and imagery tasks are reported elsewhere). About half of the participants performed the present haptic tasks in the morning, and other tasks in the afternoon. For the other half, the ordering was the other way around. Thirteen early-blind, 17 late-blind, and 16 sighted individuals participated in the present experiments (see Table 1). The blind were recruited via announcements in magazines for the visually impaired. The sighted participants had blind partners or relatives, or worked (on a paid or voluntary basis) in institutions for the blind. None of the participants had neurological or motor deficits, and none of the early blind reported any memory of vision. All participants in the late-blind group had rich, vivid visual memories and reported having used vision as a primary spatial modality. The sighted control participants were blindfolded.

The early-blind, late-blind, and sighted control participants were matched for $\operatorname{sex}\left[\chi^{2}(2)=1.1, p=.57\right]$ and verbal IQ, which was assessed via two subscales (Vocabulary and Similarities) of the Dutch WAIS-III (Wechsler, 1997) $[F(2,43)=1.4, p=.3]$. Furthermore, the groups were (approximately) matched for age $[F(2,43)=2.8$, $p=.071]$ and education $[F(2,43)=3 \cdot 2, p=.05]$. Education level was coded on a 5-point scale. The participants were right-handed, as assessed with Annett's (1970) handedness questionnaire, except as indicated in Table 1.

All participants gave their informed consent for inclusion in this study and received payment for their participation. They were naive to all aspects of the tasks; that is, they had never seen or felt the setup, were unaware of the experimental purposes, and were never given any feedback.

Apparatus and Stimuli. See Figure 1. The participants were seated $3-5 \mathrm{~cm}$ from the middle of the long end of a table with an iron top. The stimuli were two aluminum bars, of length $20 \mathrm{~cm}$ and diameter $1.1 \mathrm{~cm}$, presented on two protractors $(20 \mathrm{~cm}$ in diameter), indicated by circles in Figure 1. The centers of these protractors were $20.5 \mathrm{~cm}$ from the table's edge. The bars were $120 \mathrm{~cm}$ apart; this distance is known to yield relatively large egocentric errors, thus allowing ample opportunity for any allocentric influence to be demonstrated (Kappers, 1999). The aluminum bars could be rotated around the center of the protractor on a small pin attached to the middle of each bar, which fitted exactly into a hole in the center of the protractor. The bars tapered off at both ends, allowing the experimenter to accurately read their orientations (uncertainty of about $0.5^{\circ}$ ). Small magnets fixed to the bars were used to increase the resistance to movement. Each of the bars was used as both a reference and a test bar in different trials. In each trial, the test bar was to be matched to the reference bar's orientation by rotating it with the corresponding hand - that is, the bar to the left of the body was rotated/ explored with the left hand, and the one to the right with the right hand. The reference bar was presented in one of six orientations, in order to provide variation: $0^{\circ}, 30^{\circ}, 60^{\circ}, 90^{\circ}, 120^{\circ}$, and $150^{\circ}$ (with $0^{\circ}$ being parallel to the long side of the table, and increasing values signifying rotation in a counterclockwise direction). The test bar was presented in a random orientation-that is, corresponding to the orientation of the second hand of a clock at that particular time. In both conditions, each reference bar orientation was presented three times at each of the bar locations, for a total of 36 trials per condi- tion. The order of reference bar presentation was randomized over participants, and an Apple Macintosh computer was used to provide time restriction signals.

Procedure. Before the actual experiment, the participants were presented with an extensive preexperimental session to make sure they had a correct understanding of parallelity. They were asked to sit down at the stimulus presentation table, which was covered by cloths concealing 15 bars. The sighted participants were blindfolded. The participants were told that they were sitting at a table on which lay several bars. They were asked if they were familiar with the term parallel, and all participants reported that they were. The following definition of parallelism was given: "Bars are parallel when they never meet or cross, when elongated infinitely." As examples of parallel lines, railroad tracks were mentioned, as well as the opposite sides of a rectangle. In addition, participants were asked to come up with an example for themselves. Furthermore, they were given two pens and were asked to lay them parallel to each other on the table. The participants were then asked to rotate all 15 bars on the table at which they were sitting parallel to the bar right in front of them as accurately as possible, using the right hand only, while remaining seated on the stool with their navel in front of the middle of the long end of the table. They were freely allowed to choose their strategy and to take as long as they wanted within $10 \mathrm{~min}$, which proved a more than sufficient amount of time for all participants. These extensive instructions ensured that all participants expressed an appropriate understanding of parallelity.

After this preexperimental parallelity training/assessment session, the participants were told that at this point all bars would be removed, except two. The experimenter then took a participant's hands and guided them to the remaining bars. The participants were instructed that they would repeatedly have to feel a reference orientation and then rotate a test bar in such a way that they thought it was parallel to the just-felt reference bar orientation, either with or without a delay. The timing of the sequence of required actions was carried out by a computer: A sampled male voice uttered the numbers "one" (signifying the start of exploration), "two" (1 sec later, marking the end of exploration and the start of either the parallel-setting or the delay), "three" (either the end of parallel-setting - $1.4 \mathrm{sec}$ later - or the end of delay/start of parallel-setting-10 sec later), and in the 10-sec delay condition, also the number "four" (signifying the end of parallel-setting - $1.4 \mathrm{sec}$ later). The time restrictions for exploration, delay, and response were based on the findings of Zuidhoek et al. (2003).

Five practice trials were performed to ensure that the instructions had been understood correctly and to get the participants used to the timed sequence in which the actions had to be performed. In each trial, the experimenter positioned the reference bar in a predetermined orientation and the test bar in a random orientation. Next, he took the hand that was to explore the reference bar in that particular trial, saying (the Dutch equivalent of " "Here is the reference bar," and left the hand floating about $5 \mathrm{~cm}$ above the reference bar. He then took the participant's other hand, saying "Here is the test bar," and left the hand floating about $5 \mathrm{~cm}$ above the test bar. After this, the experimenter started the timed sequence.

The participants had to stick to the time restrictions when they explored the reference bar with one hand, waited, or rotated the test bar with the other. In the exploration and response phases, the participants were free to choose their strategy, as long as they did not change the orientation of the reference bar, did not touch the edges of the table, and remained seated. After exploring the reference bar, the reference hand had to be returned to the body without touching the edge of the table. After the participant had finished the trial, the experimenter noted the orientation of the test bar and positioned the bars for the next trial.

Each participant was instructed to do the parallel-setting as accurately as possible and to keep up with the timed number signals. Occasionally, $1.4 \mathrm{sec}$ turned out to be too narrow a time window in which to perform the parallel-setting action. These trials (approximately $5 \%-10 \%$ of the total) were discarded and presented again at a later time during the session. The time to complete a condition was 
Table 1

Description of Participants

\begin{tabular}{|c|c|c|c|c|c|c|}
\hline Participant & Occupation & Education Level & Sex & Age & $\begin{array}{c}\text { Etiology of Blindness and Other } \\
\text { Characteristics }\end{array}$ & $\begin{array}{l}\text { Age at Onset } \\
\text { of Blindness } \\
\text { (Years) }\end{array}$ \\
\hline \multicolumn{7}{|c|}{ I. Early Blind } \\
\hline 1 & Sports masseuse & 3; Sec. school type 2 & $\mathrm{~F}$ & 41 & $\begin{array}{l}\text { Leber's amarosis, diffuse light } \\
\text { perception; ambidextrous }\end{array}$ & 0 \\
\hline 2 & Policy worker & 5; University & M & 41 & Retino blastoma & 0 \\
\hline 3 & Computer programmer & 5 ; Higher education & M & 33 & Macular degeneration & 0 \\
\hline 4 & Office assistant; nurse & 4; Vocational education & $\mathrm{F}$ & 49 & Rubella (mother) & 0 \\
\hline 5 & Retired operator & $\begin{array}{l}\text { 2; Sec. school type } 2 \text {, } \\
\text { unfinished }\end{array}$ & M & 58 & Glaucoma; ambidextrous & 2 \\
\hline 6 & Office assistant & 4; Sec. school type 4 & $\mathrm{~F}$ & 34 & Retrolental fibroplasias & 0 \\
\hline 7 & Operator & $\begin{array}{l}2 \text {; Sec. school type } 1 \text {, } \\
\text { unfinished }\end{array}$ & M & 38 & $\begin{array}{l}\text { Rubella (mother), diffuse light } \\
\text { perception }\end{array}$ & $2-3$ \\
\hline 8 & Interpreter/translator & 5; Higher education & $\mathrm{F}$ & 30 & Retrolental fibroplasias & 0 \\
\hline 9 & Retired & 4; Vocational education & M & 64 & Retina blastoma & $2-3$ \\
\hline 10 & Teacher; consultant & $5 ;$ Higher education & M & 46 & Leber's amarosis & 0 \\
\hline 11 & Systems designer & 5; Higher education & M & 46 & Retina blastoma & $0-1$ \\
\hline 12 & Consultant & 4; Sec. school type 4 & $\mathrm{~F}$ & 55 & Retina blastoma & $0-1$ \\
\hline 13 & Sound technician & 5 ; Higher education & M & 49 & Retrolental fibroplasias & 0 \\
\hline \multicolumn{7}{|c|}{ II. Late Blind } \\
\hline 1 & Unemployed physiotherapist & 5; Higher education & $\mathrm{M}$ & 52 & Accident, diffuse light perception & 10 \\
\hline 2 & IT employee & 5 ; Higher education & M & 57 & $\begin{array}{l}\text { Born partially sighted and blind in one } \\
\text { eye; other eye: glaucoma }\end{array}$ & 25 \\
\hline 3 & Social worker & 4; Vocational education & M & 57 & $\begin{array}{l}\text { One eye lost at age } 21 \text { from Usher's } \\
\text { syndrome, other eye damaged in } \\
\text { accident; additional hearing problems } \\
\text { (left ear } 30 \% \text { hearing) }\end{array}$ & 25 \\
\hline 4 & Piano tuner & 4; Vocational education & M & 40 & Accident; left-handed & 19 \\
\hline 5 & Volunteer & 5 ; Higher education & M & 54 & Macular degeneration & \pm 10 \\
\hline 6 & Office assistant & 3; Sec. school type 1 & M & 64 & Congenital glaucoma & 7 \\
\hline 7 & Operator; office assistant & 3; Sec. school type 2 & $\mathrm{~F}$ & 59 & $\begin{array}{l}\text { One eye lost at age of } 2 \text { from accident; } \\
\text { second eye also lost in an accident }\end{array}$ & 9 \\
\hline 8 & Music teacher & 5 ; Higher education & M & 64 & Retinitis pigmentosa & 40 \\
\hline 9 & Correspondence clerk & 4; Vocational education & M & 60 & Congenital glaucoma & 49 \\
\hline 10 & Civil servant; director & 5 ; Higher education & M & 38 & Brain tumor & 4 \\
\hline 11 & $\begin{array}{l}\text { Employment consultant for the } \\
\text { blind }\end{array}$ & 5; Higher education & M & 59 & Leber opticus artrosa and glaucoma & 32 \\
\hline 12 & Social worker & 4; Vocational education & M & 53 & Aniridi and glaucoma & \pm 20 \\
\hline 13 & Operator & 4; Vocational education & $\mathrm{F}$ & 58 & $\begin{array}{l}\text { Retinitis pigmentosa, diffuse light } \\
\text { perception }\end{array}$ & 14 \\
\hline 14 & Therapist & 3; Sec. school type 1 & $\mathrm{~F}$ & 52 & $\begin{array}{l}\text { Born blind in one eye; glaucoma and } \\
\text { inflammation of the cornea of the } \\
\text { relatively good eye }\end{array}$ & \pm 30 \\
\hline 15 & School teacher & 5; Higher education & $\mathrm{F}$ & 53 & Congenital glaucoma & 22 \\
\hline 16 & Psychologist & 5; University & $\mathrm{F}$ & 51 & Congenital glaucoma & 40 \\
\hline 17 & Social worker & 5; Higher education & $\mathrm{F}$ & 39 & Unknown & 35 \\
\hline
\end{tabular}

about $20 \mathrm{~min}$ for the immediate condition and about $25 \mathrm{~min}$ for the delayed condition.

Data analysis. Studies on haptic parallelity typically compute mean signed errors, with a positive value (in degrees) signifying how far the bar to the right has been rotated clockwise with respect to the bar to the left (i.e., systematic errors), and a negative value expressing a counterclockwise (nonsystematic) error. In the present study, a relatively high number of very large errors (larger than $90^{\circ}$ in the systematic direction) occurred. Unfortunately, since the bars have arrows at both sides, deviations larger than $90^{\circ}$ have to be coded as $180^{\circ}$-opposite deviations, and thus appear large but negative in the opposite direction. For example, an error of $100^{\circ}$ would be coded as a deviation of $-80^{\circ}$. However, since most errors were in the systematic direction, a case could be made for correcting cases like the former example. Rather than doing this, in the present study we turned to circular statistics (Batschelet, 1981). Using circular sta- tistics prevents us from having to decide whether a deviation should be "corrected" or not. This method takes the deviations as they were measured and does not distinguish between $-80^{\circ}$ and $100^{\circ}$ (or more precisely, between orientations $\alpha$ and $\alpha+180^{\circ}$ ). Subsequently, this $180^{\circ}$ range of orientations that can be distinguished is normalized to the full range of $360^{\circ}$ by multiplying by 2 . The mean deviation over the six replications of every presented orientation (three for each of the two test bar positions) is determined as the mean vector sum of the normalized orientation vectors. In order to cancel the effect of doubling, the obtained orientation is then divided by 2 . The final outcome consists of a vector with a length and a direction, which is just what we need for the average deviation. To this direction we may add or subtract $180^{\circ}$, since the method cannot distinguish between directions $180^{\circ}$ apart. However, from previous studies, we know how to choose between these two possibilities. The length of the vector is a measure of the variability. 
Table 1 (Continued)

\begin{tabular}{|c|c|c|c|c|c|c|}
\hline Participant & Occupation & Education Level & Sex & Age & $\begin{array}{c}\text { Etiology of Blindness and Other } \\
\text { Characteristics }\end{array}$ & $\begin{array}{l}\text { Age at Onse } \\
\text { of Blindness } \\
\text { (Years) }\end{array}$ \\
\hline \multicolumn{7}{|c|}{ III. Sighted Controls } \\
\hline 1 & Editor & 5; Higher education & $\mathrm{F}$ & 32 & & \\
\hline 2 & Editor & 5; University & $\mathrm{F}$ & 30 & & \\
\hline 3 & Retired teacher of mathematics & 5; University & M & 58 & & \\
\hline 4 & $\begin{array}{l}\text { Assistant, research \& develop- } \\
\text { ment, department for the visually } \\
\text { impaired }\end{array}$ & 5; University & M & 37 & & \\
\hline 5 & Retired secretary & 4; Vocational education & $\mathrm{F}$ & 58 & & \\
\hline 6 & Ergotherapist & 5; Higher education & $\mathrm{F}$ & 36 & & \\
\hline 7 & Ergotherapist & 5; Higher education & $\mathrm{F}$ & 46 & & \\
\hline 8 & Freelance journalist & 5; Higher education & $\mathrm{M}$ & 56 & & \\
\hline 9 & Musician & 5; Higher education & $\mathrm{M}$ & 53 & & \\
\hline 10 & Volunteer & 4; Vocational education & $\mathrm{F}$ & 60 & & \\
\hline 11 & $\begin{array}{l}\text { Personnel \& development } \\
\text { coordinator }\end{array}$ & 5; Higher education & $\mathrm{F}$ & 54 & & \\
\hline 12 & Administration, commerce & 5; Higher education & $\mathrm{M}$ & 67 & & \\
\hline 13 & Housewife & 4; Sec. education & $\mathrm{F}$ & 63 & & \\
\hline 14 & Pedagogical trainer & 5; University & $\mathrm{F}$ & 40 & & \\
\hline 15 & Customer service manager & 5; Higher education & $\mathrm{M}$ & 48 & & \\
\hline 16 & Editor, freelance reviewer & 5; University & $\mathrm{M}$ & 51 & & \\
\hline
\end{tabular}

Note-Secondary school types: (1) 4 years, learning a trade; (2) 4 years, in preparation for secondary vocational education (administration/commerce/care); (3) 5 years, in preparation for higher education; (4) 6 years, in preparation for university.

The orientation of the reference bar was analyzed as a two-level factor, with $0^{\circ}$ and $90^{\circ}$ constituting the cardinal orientations, and $30^{\circ}$, $60^{\circ}, 120^{\circ}$, and $150^{\circ}$ the oblique orientations.

Since we were interested in the effects of both visual and haptic experience, as well as possible interaction effects, we planned comparisons for all group combinations when addressing the theoretical issues raised in the introduction. In analyses of secondary interest or of explorative character, the three-level between-subjects factor of experimental group was the default.

\section{Results}

Deviation size analyses. A 2 (delay) $\times 2$ (orientation) $\times 3$ (group) mixed ANOVA showed significant main effects of delay $[F(1,43)=12.3, p=.001]$ and orientation $[F(1,43)=5.1, p=.03]$ on deviation size. The main effect of delay signified an improvement with delayed performance, as compared with immediate parallel-setting,

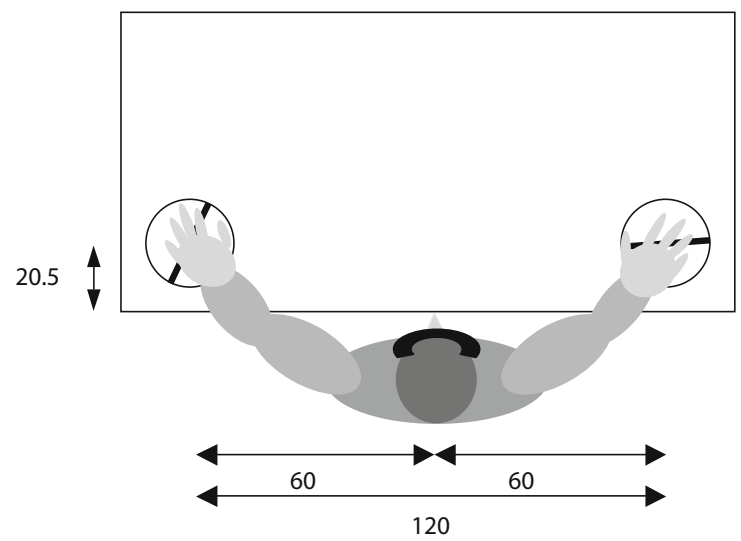

Figure 1. Top view of the experimental setup used in the parallel-setting task. with mean signed error reduced from $36.3^{\circ}$ to $28.8^{\circ}$. The main effect of orientation was expressed in different mean deviation sizes for the cardinal and oblique orientations: $29.7^{\circ}$ versus $35.3^{\circ}$, respectively.

Importantly, whereas no significant main effect of group was found $[F(2,43)=1.6, p=.22]$, nor a group $\times$ orientation interaction, there was a significant group $\times$ delay interaction $[F(2,43)=4.2, p=.022]$. Further examination of this interaction revealed that the groups differed with respect to the effect of delay (see Figure 2): Whereas delay did not affect performance for the early-blind group [38.5 and $38.8^{\circ}$ for the immediate and delayed conditions, respectively; $F(1,12)=0.002]$, it did improve performance in the late-blind $\left[36.1^{\circ}\right.$ vs. $\left.28.6^{\circ} ; F(1,16)=35.5, p<.001\right]$ and sighted control $\left[34.2^{\circ}\right.$ vs. $18.9^{\circ} ; F(1,15)=24.3, p<$ .001] groups. Furthermore, this interaction signified that performance differed among the various groups in the delayed condition $[F(2,43)=3.5, p=.04]$, whereas it did not in the immediate condition $[F(2,43)=0.2]$. Comparing late-blind and sighted performance, using a 2 (delay) $\times 2$ (orientation) $\times 2$ (group) mixed ANOVA, showed that the sighted improved significantly more with delay than did the late blind $[F(1,31)=5.6, p=.024]$.

Variability analyses. A 2 (delay) $\times 2$ (orientation) $\times$ 3 (group) mixed ANOVA showed a significant main group effect on the variability of performance $[F(2,43)=4.8$, $p=.013]$, which is expressed by the length of the mean vector $(0.77,0.88$, and 0.86 for the early-blind, late-blind, and sighted participants, respectively). Closer examination of this effect showed a significantly larger variability (indicated by a smaller mean vector) for early-blind than for late-blind $[F(1,28)=6.3, p=.02]$ and sighted $[F(1,27)=4.3, p<.05]$ participants.

A main effect was also obtained for delay $[F(1,43)=$ $10.5, p=.002]$, signifying greater variability in immedi- 


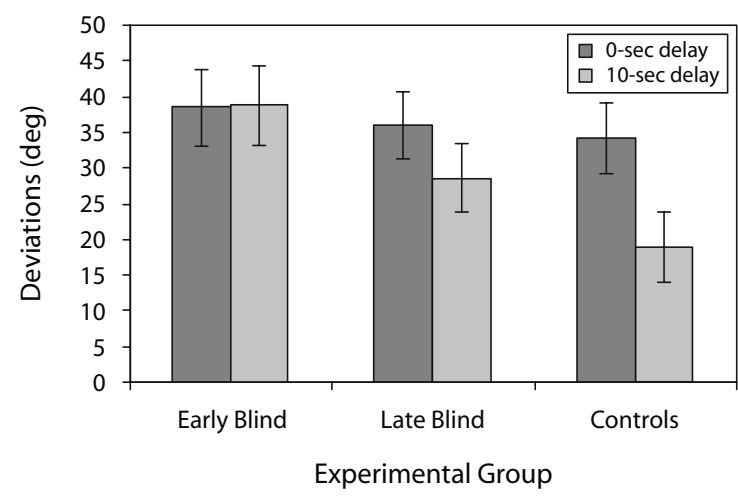

Figure 2. The interaction effect of group $\times$ delay on deviation size in the parallel-setting task. Error bars show \pm 1.0 standard errors of the means.

ate than in delayed parallel-setting, expressed by mean vector lengths of 0.82 and 0.85 , respectively. A main effect of orientation $[F(1,43)=5.7, p=.02]$ indicated that variability was lower in the cardinal than in the oblique orientations (mean vector length 0.85 vs. 0.82 , respectively). A trend was found for a group $\times$ orientation interaction $[F(2,43)=2.97, p=.06]$. Closer examination suggested that differences in variability between the cardinal and oblique orientations were clearest in the sighted group $[F(1,15)=4.96, p=.042]$. In the early blind, the orientation effect was marginally significant $[F(1,12)=$ $3.3, p=.096]$, whereas it was nonsignificant in the late blind $[F(1,16)=0.62, p=.4]$.

\section{Discussion}

The present experiment showed important commonalities and differences between early-blind, late-blind, and sighted individuals on the haptic parallel-setting task. Let us first focus on the commonalities in the performance of the three groups. The results revealed that in immediate haptic parallel-setting, early-blind, late-blind, and sighted individuals produced comparable errors with respect to size and direction, suggesting similar use of reference frames. Interestingly, both deviation sizes and variability were lower for the cardinal orientations (i.e., $0^{\circ}$ and $90^{\circ}$ ) than for the more oblique orientations. For the former orientations, this effect was independent of (early) visual experience (see also the General Discussion for more on the "oblique effect").

The present experiment also revealed three important differences between the early-blind, late-blind, and sighted groups. First, whereas the deviation size in immediate performance was comparable for the three groups, differences emerged when the parallel-setting action was delayed: Whereas delay did not affect early-blind performance, it improved late-blind and sighted performance. An improvement after a delay has been interpreted as reflecting a shift from predominantly egocentric to more allocentric reference frame involvement over time (Zuidhoek et al., 2003). The present findings thus suggest that a specific mechanism set up by early visual input is responsible for this shift in reference frame (Rossetti et al., 1996). Second, within the visually experienced groups, the sighted control group improved more with delay than did the late-blind group. Apparently, the ability to generate allocentric representations depends on early visual experience as well as on continuing visual inputs. Later loss of vision might reduce both the use of visual strategies and their efficiency in the advanced processing of haptic (orientation) information. Third, the early blind's performance was more variable than that of late-blind and sighted individuals. Early-blind individuals thus seem less consistent in generating allocentric representations on the basis of haptically perceived orientations. Notably, in all three groups, delay reduced variability.

\section{EXPERIMENT 2 Verbal Judgment-of-Orientation Task}

In the second experiment, we focused on the role of visual experience in a task designed to trigger more allocentric representations of haptically perceived orientation, even in a nondelay situation (see Zuidhoek et al., 2005). The experimental groups here assigned a number of minutes on an imagined clock (verbal response) to the haptically perceived orientation of a single bar. By explicitly defining orientation allocentrically (i.e., with respect to the stimulus presentation table) and by stimulating (visual) imagery of the bar's orientation, the task was expected to increase allocentric reference frame involvement. In addition, asking for a verbal instead of a motor response minimizes the involvement of egocentric response-related biases (i.e., distortions that may derive from perceiving and/or manipulating the response bar, as is the case with a motor response).

Indeed, a previous study on the verbal judgment of haptic orientation input revealed that deviations in the verbal judgment task were much smaller than those reported in haptic parallel-setting tasks, even when taking into account that parallel-setting comprises two bars (Zuidhoek et al., 2005): Whereas the maximum deviation in the verbal judgment study was about $1.2 \mathrm{~min}$ (or $7.2^{\circ}$ ) on average for extreme hand-midsagittal plane misalignments, haptic parallel-setting with similarly extreme misalignments has involved average deviations as large as $55^{\circ}$ or more (Kappers, 1999; Zuidhoek et al., 2004). Although small, the deviations in the verbal judgment task show the same characteristics as those from the parallel-setting task, and this result points to handcentered reference frames as the origin of the deviations in both tasks: The larger the hand-midsagittal plane misalignment, the larger the bias in perception. Given that deviations in the verbal judgment task are limited, improvements from delay cannot be expected to be very large, either. Indeed, in our previous study, only a minor delay effect (a small improvement for right-hand perceptions only) was obtained (Zuidhoek et al., 2005). Therefore, we chose not to include a delayed condition in the present verbal judgment task. 


\section{Method}

Design. The experiment had a $2 \times 2 \times 3$ factorial design, with position (ipsilateral vs. contralateral) and orientation (cardinal vs. oblique) as within-subjects factors, and group (early blind, late blind, and sighted) as a between-subjects factor. The task was the immediate verbal judgment of haptically perceived orientation (see Zuidhoek et al., 2005)

Participants. The participants were the same as in Experiment 1 .

Apparatus and Stimuli. The experimental setup was the same as in Experiment 1. Two stimulus positions were once again used, but the placing of the bars was slightly different than in Experiment 1 (see Figure 3). The bar stimuli appeared in one of eight possible orientations, in random order. To limit the experiment's duration, we chose to have the stimuli explored unimanually by the right hand only, since our previous work indicated that performance is equally good for both hands (Zuidhoek et al., 2005). The orientations were chosen in such a way that they corresponded to whole minutes. In addition, we made sure they did not correspond to multiples of $5 \mathrm{~min}$, to prevent response biases from affecting the data. The following minute positions were presented: $1,4,8,12,16,19,23$, and 27 , corresponding respectively to orientations of $84^{\circ}, 66^{\circ}, 42^{\circ}, 18^{\circ}$, $174^{\circ}, 156^{\circ}, 132^{\circ}$, and $108^{\circ}$ (in which $0^{\circ}$ was parallel to the long side of the table, with increasing orientation values signifying rotation in a counterclockwise direction)

The total number of combinations was 16 ( 2 stimulus locations $X$ 8 orientations). All combinations were presented three times, for a total of 48 trials. The order of presentation was random and different for each participant.

Procedure. The blind or blindfolded participants were told that they were sitting at the stimulus presentation table and that they would repeatedly have to feel the orientation of a bar at different locations on the table with their right hand. They were instructed to keep their exploring limb in a natural position, to think of the bar they felt as the minute hand of a clock, and to assign a number of minutes to its orientation. The orientation was allocentrically defined - that is, with respect to the stimulus presentation table. More specifically, they were told that all "clocks" on the table were positioned in such a way that when the minute hand signified 15 or $45 \mathrm{~min}$, it was parallel to the side of the table they were sitting at. To be sure that they understood this, the participants were asked to place a pen on a table in the orientation corresponding to 15 and $45 \mathrm{~min}$. They were instructed to respond as accurately as possible, in whole minutes within a response range of 0 to $60 \mathrm{~min}$, immediately after feeling the bar. They were free to give either a 0 - to $30-\mathrm{min}$ or a 30- to 60-min response.

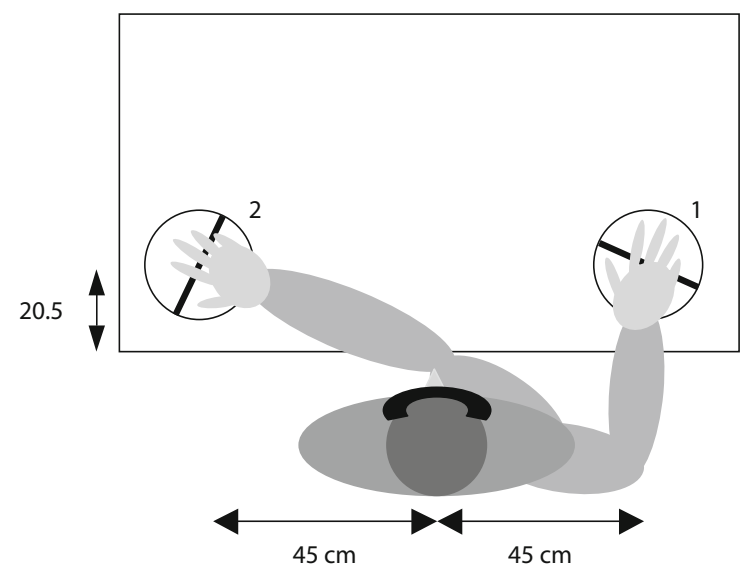

Figure 3. Top view of the experimental setup used in the verba judgment of orientation task.
Before the start of a trial, the experimenter positioned the bar in a predetermined orientation. Next, he took the participant's right hand, saying (the Dutch equivalent of) "Here is the bar," and left the hand floating about $5 \mathrm{~cm}$ above the bar. After this, the experimenter started the timed sequence on the computer. A sampled male voice uttered the numbers "one" (start of the 1-sec exploration of the bar), "two" (marking the end of exploration and the start of giving the response), and "three" (1.4 sec later, indicating the end of the response period). The time restrictions were the same as in the immediate parallel-setting task of Experiment 1.

The participants had to obey the time restrictions when exploring the bar or verbally responding. In addition, they were not allowed to touch the edges of the table during the trials and had to remain seated as they were, with respect to the table. Between trials, they were allowed to touch the table to check their positioning, which happened only occasionally. Trials in which these restrictions were not met, as checked by the experimenter, were presented again at a later time during the session (approximately $10 \%$ of the trials). Participants were free to choose their strategy when exploring the bar. In practice, they would generally just press the hand to the bar. Occasionally, this handpress was followed by a quick hand movement over the bar. After exploration of the bar, the reference hand had to be returned to the body.

Experiment 2 took about $25-30 \mathrm{~min}$ to complete.

Data analyses. The analyses were performed on signed errors, to examine the differences between the groups with respect to systematic errors. A positive value signified an overestimation of the number of minutes on the haptically perceived clock, expressing a perception of the bar's orientation as if it were rotated clockwise from its "true" orientation; a negative value signified an underestimation, expressing a perception of orientation as if the bar were rotated counterclockwise from its true orientation. Note that deviations of exactly 15 min can, in principle, be interpreted as either a large overestimation or a large underestimation. Therefore, errors of $15 \mathrm{~min}$ (only 2 of 2,208 data points) were left out of the signed-error analyses.

With respect to the haptic oblique effect, we must note that our stimuli did not contain purely horizontal $\left(0^{\circ}\right)$, vertical $\left(90^{\circ}\right)$, or "ideal" oblique $\left(45^{\circ}, 135^{\circ}\right)$ orientations, because stimulus orientations corresponding to multiples of $5 \mathrm{~min}\left(0^{\circ}\right.$ or $\left.90^{\circ}\right)$ could have led to response biases, and $45^{\circ}$ and $135^{\circ}$ do not correspond to whole minutes ( 7.5 and $22.5 \mathrm{~min}$, respectively). However, the orientations we did use deviated only a small amount from the "ideal" horizontal, vertical, and oblique orientations. Orientation of the bar therefore was analyzed as a two-level factor, with the four orientations closest to the horizontal axis (i.e., $174^{\circ}$ and $\left.18^{\circ}\right)$ and the vertical axis $\left(84^{\circ}\right.$ and $108^{\circ}$ ) forming the cardinal orientation level, and the four closest to the ideal oblique orientations $\left(42^{\circ}, 66^{\circ}, 132^{\circ}\right.$, and $\left.156^{\circ}\right)$ forming the oblique orientation level. To further examine the haptic oblique effect, we computed mean standard deviations (i.e., within-subjects standard deviations, averaged across participants, in minutes), comparing variability in the oblique and in the cardinal orientations.

Since we were interested in the effects of both visual and haptic experience, as well as possible interaction effects, we planned comparisons for all group combinations when addressing the theoretical issues raised in the introduction. In analyses of secondary interest or explorative character, the three-level between-subjects factor of experimental group was the default.

\section{Results}

Perceptual biases. A 2 (position) $\times 2$ (orientation) $\times 3$ (group) mixed ANOVA was performed on the mean signed errors. It revealed a significant main effect of position $[F(1,43)=18.7, p<.001]$, signifying a difference in performance between the two positions: The mean performance reflected an underestimation of orientation at Position 1 of $-0.77 \mathrm{~min}$ (corresponding to $4.6^{\circ}$ ), but a mean overestimation of orientation at Position 2 of $0.81 \mathrm{~min}$ (cor- 
responding to $4.9^{\circ}$ ). Orientation did not yield a significant main effect, but there was a significant interaction with position $[F(1,43)=4.99, p=.034]$. Cardinal orientations yielded a smaller undershoot than oblique ones $(-0.57$ vs. -1.56 ) for Position 1, whereas they also yielded a smaller overshoot (1.12 vs. 1.49) for Position 2.

Figure 4 depicts the performance of all three experimental groups with respect to deviations and their dependence on spatial position. With deviations averaged over the two positions being close to 0 for all groups, no significant main effect of group was found $[F(2,43)=0.66]$. However, a trend was found for a group $\times$ position interaction $[F(2,43)=2.8, p=.07]$, pointing toward larger and more systematic errors for the blind (see Figure 4): The early blind, the late blind, and the sighted controls produced the following deviations (in minutes) in verbal judgments on Positions 1 and 2, respectively: early blind, -1.3 and 1.3 ; late blind, -1.0 and 0.6 ; controls, 0.02 and 0.49 . To control for the fact that the hand's preferred orientation differed for the two positions - resulting in general overestimations in Position 2, but underestimations in Position 1 - the scores in Position 1 were multiplied by -1 , turning most of them into positive values and making them directly comparable to the scores in Position 2. A univariate mixed ANOVA was performed, with group as a two-level, between-subjects variable. Importantly, the blind (early and late blind) made significantly larger errors than the sighted $[1.3,0.8$, and 0.24 , for the early blind, late blind, and sighted, respectively; $F(1,44)=4.5$, $p<.05$ ]. Comparing the visually experienced (late blind and sighted) to the visually inexperienced (early blind) resulted in a trend toward larger errors among the early blind $[F(1,44)=3.5, p=.068]$.

Haptic oblique effect. A 2 (position) $\times 2$ (orientation) $\times 3$ (group) mixed ANOVA was performed on mean standard deviations. It revealed significant main effects of orientation $[F(1,43)=7.1, p=.011]$ as well as position $[F(1,43)=4.2, p=.046]$. The effect of orientation signified a smaller mean standard deviation for the cardinal bar orientations (close to $0^{\circ}$ and $90^{\circ}$ ) than for the more oblique ones: 2.0 versus $2.2 \mathrm{~min}$. The effect of position reflected a smaller mean standard deviation on Position 1 than on Position 2: 2.0 versus $2.2 \mathrm{~min}$, respectively.

No main or interaction effects for group were found, either from the present mixed ANOVAs or from similar ones after regroupings, with group as a two-level, betweensubjects factors. In addition, no effect of position $\times$ orientation type was found $[F(1,43)=0.1]$, suggesting that the haptic oblique effect was independent of the location of the perceived bar.

\section{Discussion}

The results show that the haptic perception of singlebar orientation is systematically biased in blind as well as in sighted individuals. The directions and sizes of the mean errors on both positions were consistent with those of a previous study, pointing toward a prominent role of a hand-centered reference frame (Zuidhoek et al., 2005). Most importantly, once again, deviations appeared to be more pronounced in the blind, with the late blind seeming

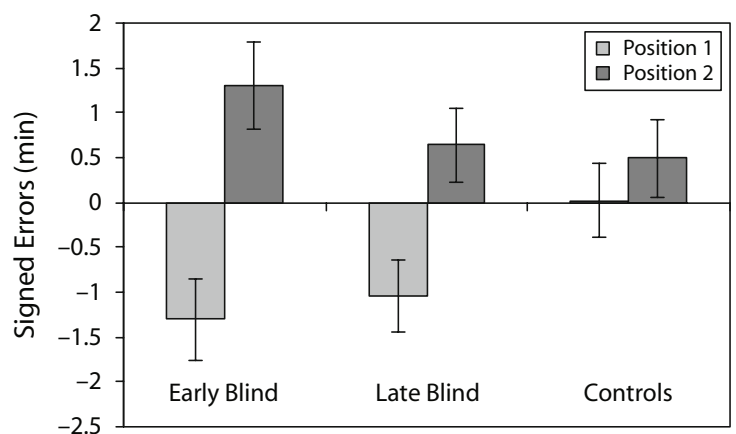

Experimental Group

Figure 4. Performance of early-blind, late-blind, and sighted participants on Positions 1 and 2 in the verbal judgment task. Error bars show \pm 1.0 standard errors of the means.

to perform more like the early blind than like the sighted. Interestingly, participants again seemed to perform better at the cardinal orientations (i.e., close to $0^{\circ}$ and $90^{\circ}$ ) than at the more oblique ones, irrespective of their visual or haptic experience. This was reflected in the variability in performance, which is known as the oblique effect.

We may speculate here that the systematic bias in perception and the haptic oblique effect result from different neurocognitive mechanisms, since they were affected differently by location in space and experimental group. Whereas the systematic bias in perception and its variability (standard deviation) both depended on the location of the bar in space, the haptic oblique effect (the difference in variability for horizontal and vertical orientations as compared with oblique orientations) did not (see Zuidhoek et al., 2005). Furthermore, although being blind resulted in slightly larger systematic errors in the haptic perception of single-bar orientation, blindness did not affect the haptic oblique effect.

\section{GENERAL DISCUSSION}

The main goal of the present study was to investigate the role of visual experience in the haptic perception of orientation in peripersonal space. To this end, early-blind, late-blind, and sighted participants were presented with two tasks requiring allocentric representations in order to be performed successfully. Performance differences between these groups can shed light on the mechanisms involved in haptic orientation perception and help to evaluate some of the leading ideas on the effect of blindness on spatial representation for haptic spatial perception in peripersonal space. In the first experiment, a haptic matching task, a test bar had to be rotated until it was parallel to a reference bar in immediate and 10-sec delay conditions. In the second experiment, instead of a motor response, a verbal response was required: Participants had to assign a number of minutes on an imagined clock to the orientation of a single bar, with orientation explicitly defined with respect to an allocentric reference framenamely, the stimulus table. 
In general, the results of the present study suggest that visual experience helps in certain aspects of haptic orientation processing. Immediate motor reproduction in the parallel-setting task may cause participants of all experimental groups to act on an egocentrically biased haptic perception of the bars' orientations - that is, they may act on what they haptically perceive, without addressing other mechanisms to represent the bars. In contrast, a delay may stimulate visually experienced participants to turn to other, arguably visual, mechanisms to retain the haptic orientation information. In turn, this may result in recoding of the haptic information in more allocentric reference frames (see Rossetti et al., 1996). This line of reasoning follows from several clear group differences that we observed. First, the early blind did not improve with delay, whereas the late blind did, but to a lesser degree than the sighted. Also, the early blind performed more variably than lateblind and sighted participants, suggesting that early visual experience may provide structure to the representations derived from haptic inputs (i.e., make performance more reliable and consistent). Furthermore, on the verbal judgment task, which can be argued to evoke a more explicit definition of the bar orientation with respect to an allocentric reference frame (i.e., the stimulus table), both the early and late blind performed worse than the sighted. Together, it seems that differences between the early blind, late blind, and sighted on allocentric haptic perception tasks may particularly emerge in tasks that induce participants to abandon representations at the haptic or motor level.

Having established that visual mechanisms play a role in haptic spatial perception, the obvious next question concerns the nature of these mechanisms. A likely mechanism would be visuospatial imagery; that is, haptic information must be converted to a mental image that is independent of the primary receptors and their orientations (i.e., the hands). This mental image can guide behavior (i.e., the motor responses in the delayed parallel-setting task), and it allows for conscious inspection and interpretation (i.e., the verbal judgments in Experiment 2). Whereas the literature has clearly established that blind individuals can work with mental images (Kaski, 2002), there are also a number of quantitative and qualitative differences between their performance and that of sighted persons (see Aleman, van Lee, Mantione, Verkoijen, \& de Haan, 2001; Noordzij, Zuidhoek, \& Postma, 2007). Cornoldi and Vecchi (2003) concluded, on the basis of several studies on visuospatial working memory, that congenitally blind individuals specifically experience problems with the generation, maintenance, and manipulation of multiple mental images. Although this may explain differences between early-blind and sighted participants on the imagery condition (i.e., the delayed condition) of the parallelsetting task (which requires the formation of two images), it does not fit with the group differences observed on the verbal judgment task (where only a single image is necessary). Thinus-Blanc and Gaunet (1997) offered a slightly different view on imagery in the blind. They argued that "the consequence of an early lack of vision would be that (1) the amount of information stored in the form of mental images is diminished (or lacking) and (2) complex compu- tations that rely on such types of representations are more difficult" (p. 24). This view may explain the greater performance variability in the blind, from diminished quality of the images. In addition, the verbal judgment task might be considered to involve more complex imagery, on which even participants with early but no recent visual experience (i.e., the late blind) underachieve.

An alternative account of some of the results discussed above could be that the delayed condition in Experiment 1 yielded its effects not because it caused a recoding of the haptic information, but simply because it allowed more time for the required processes to be satisfactorily completed. In particular, the initial coding time may have been too strict $(1 \mathrm{sec})$. However, whereas elsewhere we indeed observed an advantage with longer exploration times (i.e., $5 \mathrm{sec}$ ), this advantage was independent of variation in the length of the subsequent retention interval (Zuidhoek et al., 2003). Moreover, restricted exploration time cannot easily explain the performance differences between blind and sighted individuals.

The haptic oblique effect - that is, lower variability on horizontal and vertical orientations than on oblique orientations - sheds further light on the mechanisms involved in haptic processing of spatial orientations. Appelle and colleagues (Appelle \& Countryman, 1986; Appelle \& Gravetter, 1985) found that the oblique effect was eliminated when, prior to testing, no visual or verbal knowledge about the stimulus orientations was conveyed to the participants. Appelle and colleagues therefore suggested that visual processing is critically responsible for the oblique effect. However, a more recent study of the effect of early visual experience on the haptic oblique effect (Gentaz \& Hatwell, 1998), using a motor reproduction response, did not support this interpretation: There, early- as well as late-blind participants displayed the haptic oblique effect, and did so to the same extent. The present findings partly replicate those reported by Gentaz and Hatwell (1998): In our Experiment 2, oblique effects did not differ between the groups, whereas in Experiment 1 there was a trend for the oblique effect to be most evident in the sighted group. Together, the present results suggest that the preference for some orientations over others that we observed in both variability (i.e., the oblique effect) and deviation size may, for the greater part, be independent of response mode, location in space, and visual experience.

To conclude, the present findings offer important insights into how we interpret what we feel with our hands and how this is guided by concurrent visual processing mechanisms. A motor reproduction of haptically perceived stimulus orientation might strongly engage an egocentric or body-related frame of reference. Delaying the response or asking for a verbal response seems to evoke a shift toward a more allocentric representation, possibly stimulated by forming an explicit mental image of the felt orientation. Under these circumstances, visual experience becomes critically important, as differences between the early and late blind, on one hand, and sighted individuals, on the other, attest. Visual experience may exert its role either by offering the opportunity to use relevant stored visual images (see Thinus-Blanc \& Gaunet, 1997) or by 
increasing the tendency or ability to employ visual imagery (see Ernest, 1987). As such, the present findings suggest that the ability to recode haptic spatial input into visuospatial images could be an important tool for improving the allocentric representations required in certain haptic spatial tasks.

\section{AUTHOR NOTE}

The authors thank Christine Bijl for her help in conducting the experiments and two anonymous referees for their constructive comments. This research was supported by the Netherlands Organization for Scientific Research (Grants NWO 440-20-000 and NWO 016-048-606) and EU NEST Fp6 (Grant 12959, Wayfinding). Correspondence related to this article may be sent to A. Postma, Experimental Psychology, Utrecht University, Heidelberglaan 2, 3584 CS Utrecht, The Netherlands (e-mail: a.postma@uu.nl).

\section{REFERENCES}

Aleman, A., van Lee, L., Mantione, M. H. M., Verkoijen, I. G., \& DE HAAN, E. H. F. (2001). Visual imagery without visual experience: Evidence from congenitally totally blind people. NeuroReport, 12 , 2601-2604

AnNetT, M. (1970). A classification of hand preference by association analysis. British Journal of Psychology, 61, 303-321.

ApPelle, S., \& Countryman, M. (1986). Eliminating the haptic oblique effect: Influence of scanning incongruity and prior knowledge of the standards. Perception, 15, 325-329.

Appelle, S., \& Gravetter, F. (1985). Effect of modality-specific experience on visual and haptic judgment of orientation. Perception, 14, 763-773.

Batschelet, E. (1981). Circular statistics in biology. London: Academic Press.

Bridgeman, B., Peery, S., \& Anand, S. (1997). Interaction of cognitive and sensorimotor maps of visual space. Perception \& Psychophysics, 59, 456-469.

CoRNOLdi, C., \& VeCCHI, T. (2003). Visuo-spatial working memory and individual differences. Hove, U.K.: Psychology Press.

ERNEST, C. H. (1987). Imagery and memory in the blind: A review. In M. A. McDaniel \& M. Pressley (Eds.), Imagery and related mnemonic processes: Theories, individual differences, and applications (pp. 218-238). New York: Springer.

Gentaz, E., \& Hatwell, Y. (1998). The haptic oblique effect in the perception of rod orientation by blind adults. Perception \& Psychophysics, 60, 157-167.

GentaZ, E., \& Hatwell, Y. (1999). Role of memorization conditions in the haptic processing of orientations and the "oblique effect." British Journal of Psychology, 90, 373-388.

Heller, M. A., Calcaterra, J. A., Green, S. L., \& Barnette, S. L. (1999). Perception of the horizontal and vertical in tangible displays: Minimal gender differences. Perception, 28, 387-394.

Kappers, A. M. L. (1999). Large systematic deviations in the haptic perception of parallelity. Perception, 28, 1001-1012.

KAPPERS, A. M. L. (2002). Haptic perception of parallelity in the midsagittal plane. Acta Psychologica, 109, 25-40.

KAPPERS, A. M. L. (2003). Large systematic deviations in a bimanual parallelity task: Further analysis of contributing factors. Acta Psychologica, 114, 131-145.

KAPPERS, A. M. L., \& KoENderinK, J. J. (1999). Haptic perception of spatial relations. Perception, 28, 781-795.

KASKI, D. (2002). Revision: Is visual perception a requisite for visual imagery? Perception, 31, 717-731.
LanCa, M., \& Bryant, D. J. (1995). Effect of orientation in haptic reproduction of line length. Perceptual \& Motor Skills, 80, 1291-1298.

Lederman, S. J., KlatzKy, R. L., \& Barber, P. O. (1985). Spatial and movement-based heuristics for encoding pattern information through touch. Journal of Experimental Psychology: General, 114, 33-49.

Luyat, M., Gentaz, E., Corte, T. R., \& Guerraz, M. (2001). Reference frames and haptic perception of orientation: Body and head tilt effects on the oblique effect. Perception \& Psychophysics, 63, 541-554.

Marks, L. E., \& Armstrong, L. (1996). Haptic and visual representations of space. In T. Inui \& J. L. McClelland (Eds.), Attention and performance XVI: Information integration in perception and communication (pp. 263-287). Cambridge, MA: MIT Press.

Millar, S. (1988). Models of sensory deprivation: The nature/nurture dichotomy and spatial representation in the blind. International Journal of Behavioral Development, 11, 69-87.

MiLlaR, S. (1994). Understanding and representing space: Theory and evidence from studies with blind and sighted children. Oxford: Oxford University Press, Clarendon Press.

Millar, S., \& Al-AtTaR, Z. (2004). External and body-centered frames of reference in spatial memory: Evidence from touch. Perception \& Psychophysics, 66, 51-59.

Milner, A. D., Paulignan, Y., Dijkerman, H. C., Michel, F., \& JeanNEROD, M. (1999) A paradoxical improvement of misreaching in optic ataxia: New evidence for two separate neural systems for visual localization. Proceedings of the Royal Society B, 266, 2225-2229.

NewPort, R., RABB, B., \& JACKSON, S. R. (2002). Noninformative vision improves haptic spatial perception. Current Biology, 12, 1661-1664.

Noordzis, M. L., Zuidhoek, S., \& Postma, A. (2007). The influence of visual experience on visual and spatial imagery. Perception, 36, 101-112.

PaIvio, A. (1978). Comparisons of mental clocks. Journal of Experimental Psychology: Human Perception \& Performance, 4, 61-71.

Rossetti, Y., Gaunet, F., \& Thinus-Blanc, C. (1996). Early visual experience affects memorization and spatial representation of proprioceptive targets. NeuroReport, 7, 1219-1223.

RossetTI, Y., \& RÉGNIER, C. (1995). Representations in action: Pointing to a target with various representations. In B. G. Bardy, R. J. Bootsma, \& Y. Guiard (Eds.), Studies in perception and action III (pp. 233-236). Mahwah, NJ: Erlbaum.

Thinus-Blanc, C., \& Gaunet, F. (1997). Representation of space in blind persons: Vision as a spatial sense? Psychological Bulletin, 121, 20-42.

WeCHSLER, D. (1997). WAIS-III: The Wechsler Adult Intelligence Scale (3rd ed.). San Antonio, TX: Psychological Corp.

Zuidhoek, S., Kappers, A. M. L., \& Postma, A. (2005). Effects of hand orientation and delay on the verbal judgment of haptically perceived orientation. Perception, 34, 741-755.

Zuidhoek, S., Kappers, A. M. L., van der Lubbe, R. H., \& Postma, A. (2003). Delay improves performance on a haptic spatial matching task. Experimental Brain Research, 149, 320-330.

Zuidhoek, S., Visser, A., Bredero, M. E., \& Postma, A. (2004). Multiple integration mechanisms in haptic space perception. Experimental Brain Research, 157, 265-268.

\section{NOTE}

1. It should be acknowledged here that visual perception is also open to distortions and may also code space in an egocentric way under particular circumstances. What is critical here, though, is that relative to haptic processing, vision may offer more opportunity for allocentric interpretations of space.

(Manuscript received December 23, 2004; revision accepted for publication March 18, 2008.) 